\title{
Multimodal CT Provides Improved Performance for Lacunar Infarct Detection
}

\author{
T. Das, F. Settecase, M. Boulos, T. Huynh, C.D. d'Esterre, S.P. Symons, L. Zhang, and R.I. Aviv
}

\begin{abstract}
BACKGROUND AND PURPOSE: Lacunar infarcts account for approximately $25 \%$ of acute ischemic strokes. Compared with NCCT alone, the addition of CTP improves sensitivity for detection of infarcts overall. Our aim was to systematically evaluate the diagnostic benefit and interobserver reliability of an incremental CT protocol in lacunar infarction.
\end{abstract}

MATERIALS AND METHODS: Institutional review board approval and patient consent were obtained. One hundred sixty-three patients presenting with a lacunar syndrome $\leq 4.5$ hours from symptom onset were enrolled. Images were reviewed incrementally by 2 blinded readers in 3 separate sessions (NCCT only, NCCT/CTA, and NCCT/CTA/CTP). Diagnostic confidence was recorded on a 6-point scale with DWI/ADC as a reference. Logistic regression analysis calculated differences between actual and observed diagnoses, adjusted for confidence. Predictive effects of observed diagnostic accuracy and confidence score were quantified with the entropy $r^{2}$ value. Sensitivity, specificity, and confidence intervals were calculated accounting for multiple readers. Receiver operating characteristic analyses were compared among diagnostic strategies. Interobserver agreement was established with Cohen $\kappa$ statistic.

RESULTS: The final study cohort comprised 88 patients (50\% male). DWI/ADC-confirmed lacunar infarction occurred in 59/88 (67\%) with $36 / 59(61 \%)$ demonstrating a concordant abnormal finding on CTP. Sensitivity for definite or probable presence of lacunar infarct increased significantly from $9.3 \%$ to $42.4 \%$ with incremental protocol use, though specificity was unchanged (range, 91.9\%-95.3\%). The observed diagnosis was significantly related to the actual diagnosis after adjusting for CTP confidence level $(P=.04)$ and was 5.1 and 2.4 times more likely to confirm lacunar infarct than NCCT or CTA source images. CTP area under the curve (0.77) was significantly higher than that of CTA source images $(0.68, P=.006)$ or NCCT $(0.55, P<.001)$.

CONCLUSIONS: CTP offers an improved diagnostic benefit over NCCT and CTA for the diagnosis of lacunar infarction.

ABBREVIATIONS: $\mathrm{AIC}=$ Akaike information criterion; $\mathrm{CTA}-\mathrm{SI}=\mathrm{CT}$ angiographic-source images; $\mathrm{IQR}=$ interquartile range; $\mathrm{LI}=$ lacunar infarct

$\mathbf{L}^{2}$ acunar infarcts (LIs) account for approximately $25 \%$ of all acute ischemic strokes. ${ }^{1}$ These small subcortical infarcts are typically located in the basal ganglia, thalamus, internal capsule, corona radiata, or brain stem. ${ }^{2}$ Although single perforating artery occlusion is the dominant pathophysiology, lacunar syndromes may be secondary to large-artery disease and cardioembolic

Received August 11, 2014; accepted after revision December 11.

From the Department of Radiology (T.D.), Cambridge University Hospitals NHS Foundation Trust, Addenbrooke's Hospital, Cambridge, UK; Department of Neurology (C.D.d.), University of Calgary, Calgary Stroke Program, Foothills Medical Centre, Calgary, Alberta, Canada; Departments of Medical Imaging (F.S., T.H., R.I.A., S.P.S., L.Z.) and Neurology (M.B.), Sunnybrook Health Sciences Centre, Toronto, Ontario, Canada; and University of Toronto (M.B., T.H., S.P.S., R.I.A.), Toronto, Ontario, Canada.

Paper previously presented in part as an oral presentation at: American Society of Neuroradiology Annual Meeting and the Foundation of the ASNR Symposium May 18-23, 2013; San Diego, California.

Please address correspondence to Richard I. Aviv, MD, Sunnybrook Health Sciences Centre, 2075 Bayview Ave, Room AG-31E, Toronto, ON, M4N 3M5, Canada; e-mail: Richard.aviv@sunnybrook.ca

http://dx.doi.org/10.3174/ajnr.A4255 causes in up to $10 \%$ of cases. ${ }^{3}$ The National Institutes of Neurological Disorders and Stroke study showed the benefit of thrombolytic use on functional outcome across all stroke subtypes, including small-vessel infarcts characterized at baseline. ${ }^{4}$ Although conflicting findings have been reported, ${ }^{5,6}$ a general consensus favors thrombolytic use in lacunar infarction. ${ }^{7}$ Anecdotally, thrombolysis treatment of an acute LI was associated with MR imaging perfusion and DWI deficit reversal, with good clinical recovery. ${ }^{8}$ Inaccuracy of the LI diagnosis is present in approximately $30 \%$ of stroke presentations by using clinical and NCCT findings alone. ${ }^{9}$ CTP is increasingly used during acute stroke work-up due to its cost effectiveness, ease, availability, speed, and tolerability. ${ }^{10} \mathrm{CTP}$ significantly improves sensitivity for detection of infarcts overall compared with NCCT alone. ${ }^{11,12}$ Perfusion abnormality associated with LI is clinically important and may be associated with early clinical deterioration, infarct growth, or resolution. ${ }^{8,13-15}$ No prior study has systematically evaluated the performance of CTP for LI, to our knowledge. The purpose of 
this study was to evaluate the diagnostic benefit and interobserver reliability of an incremental CT protocol in the diagnosis of LI.

\section{MATERIALS AND METHODS \\ Study Cohort}

All study procedures and medical chart reviews were approved by the local institutional research ethics board. Patients were retrospectively selected from a prospectively acquired database of consecutive patients presenting to a tertiary stroke center between March 2011 and February 2013, admitted under a code stroke designation to the emergency department $<4.5$ hours after symptom onset. Inclusion criteria were presentation with an acute code stroke designation with a clinical lacunar syndrome ${ }^{16}$ determined at admission by the attending stroke neurologists (5-10 years' experience) and undergoing the admission CT stroke protocol (NCCT, CT angiographic-source images [CTA-SI], and CTP) and follow-up MR imaging, with or without restriction in a LI distribution on DWI/ADC and no vessel occlusion. Patients with both cortical and lacunar infarcts were excluded from review ( $n=$ 44). Of 163 patients presenting with a clinical lacunar syndrome, we excluded the following: 11 patients with stroke mimic (cavernous malformation, aneurysm, venous thrombosis, seizure, migraine, 5.3\%) and 64 with delayed follow-up MR imaging (>30 days from presentation) or MR imaging performed following repatriation and not available for review (30.9\%). A 30-day DWI limit was applied, because this represents an accepted time for DWI to remain sensitive for infarct detection in patients with acute ischemic stroke. ${ }^{1,17}$ LI was defined as solitary, subcortical lesions, $\leq 20 \mathrm{~mm}$ in greatest diameter as previously defined, ${ }^{15}$ recommended, ${ }^{18}$ and clinically applied. ${ }^{19,20}$ The final study cohort comprised 88 patients.

\section{Imaging Protocol}

CT stroke protocol, performed on a 64-section CT scanner (VCT; GE Healthcare, Milwaukee, Wisconsin), includes a pre- and postcontrast head CT with the following parameters: $120 \mathrm{kV}$ (peak), $340 \mathrm{~mA}, 8 \times 5 \mathrm{~mm}$ collimation, 1 s/rotation, and table speed of 15 $\mathrm{mm} /$ rotation. CTA was performed from the aortic arch to the vertex with the following parameters: $0.7-\mathrm{mL} / \mathrm{kg}$ iodinated contrast agent up to a maximum of $90 \mathrm{~mL}$ (iohexol, Omnipaque 300 mg iodine/mL; GE Healthcare, Piscataway, New Jersey), 5- to 10second delay, $120 \mathrm{kVp}, 270 \mathrm{~mA}, 1$ s/rotation, 1.25-mm-thick sections, and table speed of $3.7 \mathrm{~mm} /$ rotation. Biphasic CT perfusion included a 45-second scan reconstructed at 0.5-second intervals followed by 6 further acquisitions 15 seconds apart for an additional 90 seconds. CTP scan parameters were the following: 80 $\mathrm{kVp}, 100 \mathrm{~mA}, 0.5-\mathrm{mL} / \mathrm{kg}$ (maximum, $50 \mathrm{~mL}$ ) iodinated contrast agent injected at $4 \mathrm{~mL} / \mathrm{s}$ with a 3 - to 5 -second delay. ${ }^{11}$ Sections were selected to cover either the infra- or supratentorium, depending on clinical suspicion of infarct location by an attending neurologist. In all cases, the first supratentorial and last infratentorial CTP sections included the inferiormost portion of the frontal horn of the lateral ventricle. Follow-up MR imaging comprised at least DWI (TR $=8125 \mathrm{~ms} /$ minimum TE, 26-cm FOV, $128 \times$ 128 matrix, 5-mm section thickness, no intersection gap) and FLAIR sequences $(\mathrm{TR}=8000 \mathrm{~ms} / \mathrm{TE}=120 \mathrm{~ms} / \mathrm{TI}=200 \mathrm{~ms}$, 22-cm FOV, $320 \times 224$ matrix, 5-mm section thickness, 1-mm intersection gap). Effective doses of individual components of CT stroke protocol were NCCT, $1.2 \mathrm{mSv}$; CTA, $2.4 \mathrm{mSv}$; and CTP, 2.5 $\mathrm{mSv}$. Lifetime attributable risk of cancer for NCCT at the median age of the included cohort is $0.01 \% .^{21}$

\section{Image Processing}

CT Perfusion 4 (GE Healthcare) was used to calculate parametric maps as described previously. ${ }^{11}$ Briefly, arterial input and venous time-attenuation curves were generated, after manually selecting regions of interest in the anterior cerebral artery ipsilateral to the side of the infarct and the superior sagittal sinus, respectively. PACS postprocessing of CTA-SI (4 mm thick with a 2-mm gap and aligned to match the NCCT) was performed by CT technologists at the CT operator console.

\section{Review Protocol}

The review protocol simulated the usual order in which stroke studies are reviewed in our practice, beginning with NCCT images, followed by CTA and CTA-SI and CTP color maps. The 2 reviewers were a recently trained neuroradiologist and an experienced neuroradiology fellow (1-years' experience in a high-volume stroke center). Before review, 3 anonymized DICOM folders were prepared and stored on a secure server. The first folder contained only NCCT images; the second, NCCT and CTA-SI; and the third, NCCT, CTA-SI, and CTP color maps (CBF, CBV, and MTT). Only 1 folder was available to a reader at a time, and each completed folder was removed following completion. A minimum 2-week interval separated each review to minimize recall bias. Images were reviewed with a Windows-based PACS (KPACS, version 1.6.0; http://www.k-pacs.de). Reviewers were blinded to all clinical information including the affected side. For each patient, the reviewers scored both hemispheres for the presence or absence of LI and assigned a 6-point level of confidence score to each side (1, stroke definitely present; 2 , stroke probably present; 3 , equivocal but leaning to positive; 4 , equivocal but leaning to negative; 5 , stroke probably absent; 6 , stroke definitely absent). For studies with positive findings, the precise location of the infarct was recorded. For CTP, color maps were evaluated initially in combination to generate a combined score. Separate scores were also assessed for each CTP map. The total number of scores generated for each technique was 352 (88 patients, 2 sides and 2 readers). The final observed diagnosis was established on DWI/ ADC coregistered to baseline imaging by an experienced neuroradiologist (9 years' experience) (SPM 8; http://www.fil.ion. ucl.ac.uk/spm/software). True-positive studies required a confidence score of 1-3 and identification of the correct infarct location. Incorrect location with scores of $1-3$ constituted a falsepositive response.

\section{Statistical Analysis}

Analyses were performed with SAS (Version 9.1; SAS Institute, Cary, North Carolina). Results were expressed as either the mean $\pm \mathrm{SD}$ or median and interquartile range (IQR) for quantitative variables and as proportions for categoric findings. Comparisons of demographic features were performed by using an unpaired samples $t$ test and Mann-Whitney $U$ and $\chi^{2}$ tests. 
Table 1: Comparison of baseline demographic features of 88 patients presenting with acute stroke symptoms with and without lacunar infarct ${ }^{\mathrm{a}}$

\begin{tabular}{lccc}
\hline & $\begin{array}{c}\text { Lacunar Infarct Present } \\
(\boldsymbol{n}=\mathbf{5 9})\end{array}$ & $\begin{array}{c}\text { Lacunar Infarct Absent } \\
(\boldsymbol{n}=\mathbf{2 9})\end{array}$ & $\boldsymbol{P}$ Value \\
\hline Mean age (yr) (SD) & $72(13.4)$ & $68(17.5)$ & .30 \\
Median NIHSS (IQR) & $6.5(5)$ & $2(5)$ & .16 \\
Median days to follow-up (IQR) & $2.5(3)$ & $2(5.5)$ & .60 \\
Male sex & $33(56 \%)$ & $11(38 \%)$ & .17 \\
Cardiovascular risk factors & & & \\
Hypertension & $39(66 \%)$ & $12(41 \%)$ & $.04^{\mathrm{b}}$ \\
Diabetes & $15(25 \%)$ & $1(3 \%)$ & $.02^{\mathrm{b}}$ \\
Atrial fibrillation & $8(14 \%)$ & $1(3 \%)$ & .26 \\
Coronary artery disease & $13(22 \%)$ & $4(14 \%)$ & .40 \\
Hyperlipidemia & $23(39 \%)$ & $6(21 \%)$ & .10 \\
Smoking & $6(10 \%)$ & $1(3 \%)$ & .27 \\
\hline
\end{tabular}

${ }^{a}$ All values are No. (\%) unless otherwise specified.

${ }^{\mathrm{b}}$ Statistically significant.

Table 2: Distribution of confidence scores for infarct presence by modality for 2 readers for a total of $\mathbf{3 5 2}$ observations

\begin{tabular}{clccc}
\hline $\begin{array}{c}\text { Confidence } \\
\text { Score }\end{array}$ & NCCT & NCCT + CTA-SI & NCCT + CTA-SI + CTP \\
\hline 1 & Definitely present & $5(1.4 \%)$ & $16(4.5 \%)$ & $42(11.9 \%)$ \\
2 & Probably present & $25(7.1 \%)$ & $26(7.4 \%)$ & $27(7.7 \%)$ \\
3 & Equivocal, possibly present & $83(23.6 \%)$ & $68(19.3 \%)$ & $18(5.1 \%)$ \\
4 & Equivocal, possibly absent & $4(1.1 \%)$ & $2(0.6 \%)$ & $18(5.1 \%)$ \\
5 & Probably absent & $78(22.2 \%)$ & $89(25.3 \%)$ & $131(37.2 \%)$ \\
6 & Definitely absent & $157(44.6 \%)$ & $151(42.9 \%)$ & $116(33.0 \%)$ \\
\hline
\end{tabular}

Logistic regression analysis was used to predict the actual from observed diagnosis for the incremental protocol, adjusting for confidence score. The combined predictive effects of the observed diagnostic performance and the confidence score in the model were quantified with the entropy $r^{2}$ value calculated as $r^{2}=\left(L_{\mathrm{O}}-\right.$ $\left.L_{\mathrm{M}}\right) / L_{\mathrm{O}}$, where $L_{\mathrm{O}}$ and $L_{\mathrm{M}}$ represent the log-likelihood (maximized-2) of the null and the fitted model, respectively. The Akaike information criterion (AIC) was calculated as AIC $=L_{M}+2 n$, (where $\mathrm{n}$ is the number of parameters) and was used to compare models among the 3 modalities. Diagnostic performance of the incremental protocols was evaluated with a receiver operating characteristic curve, and the area under the curve was compared according to DeLong et al. ${ }^{22}$

To analyze correlated data from readers, we used generalized estimating equations to assess actual from observed stroke diagnosis after adjusting for the confidence score by using a generalized linear model with a binomial distribution (logit link function). The quasilikelihood information criterion was applied to fit the models. Individual sensitivity $\left(S e_{\mathrm{i}}\right)$ and specificity $\left(S p_{\mathrm{i}}\right)$ were calculated as follows:

$$
S e=\sum_{i=1}^{n} N_{i} S e_{i} / \sum_{i=1}^{n} N_{i}
$$

and

$$
S p=\sum_{i=1}^{n} N_{i} S p_{i} / \sum_{i=1}^{n} N_{i}
$$

where $N_{\mathrm{i}}$ indicates the total number of diagnoses considered for each patient and found positive at the time the actual diagnosis was made and $n$ indicates the total number of patients. The esti- mate of sensitivity and specificity and their variances were derived. ${ }^{23}$ To compare sensitivity and specificity among the 3 modalities, we performed a regression model of the natural log of $S e_{\mathrm{i}} / S p_{\mathrm{i}}$. Interobserver agreement was calculated by the Cohen $\kappa$ statistic and considered moderate, substantial, and near-perfect $(\kappa$ values $=0.41-0.60,0.61-0.80$, and $0.81-1.00$, respectively). To test the equality of the correlated Cohen $\kappa$ statistics, we performed a weighted leastsquares approach. ${ }^{24} P<.05$ was considered significant.

\section{RESULTS}

Of 88 included patients $(n=44,50 \%$ male; cohort median age, 73.5 years; $\mathrm{IQR}=60-82$ years), acute LI was demonstrated in 59 (67\%) MR imaging studies. The median time to CTP was 150 minutes (IQR $=87-196$ minutes). The median time to follow-up MR imaging was 2 days (IQR, $1-4.25$ days). The distribution of LI was lenticulostriate, 34 (57.6\%); thalamus/posterior internal capsule, 11 (18.6\%); and pons, 14 (23.7\%). Patients with LI were more likely to have hypertension and diabetes compared with those without $(P=.04 ; P=.02$, respectively; Table 1$)$. The median NIHSS score was 6.5 (IQR $=4-9$ ) in patients with LI compared with $5(\mathrm{IQR}=2-7)$ in those without, but it did not reach significance. Sixteen patients $(18.2 \%)$ received a mean dose of $73 \pm 27 \mathrm{mg}$ of IV rtPA. Perfusion abnormality was seen in 36/59 (61\%) LIs. No significant difference in age, sex, cardiovascular risk factors, and rtPA use or side of infarct was present between patients with and without a perfusion abnormality. Patients with a perfusion abnormality had a higher NIHSS score of 7.5 (IQR = $4.8-12.0$ ) versus 4.8 (IQR $=2-7, P<.01)$ and tended to present earlier (101 minutes, IQR $=70-163$ minutes versus 168 minutes $\mathrm{IQR}=120-203$ minutes, $P=.09)$. Median mRS scores $(3, \mathrm{IQR}=$ $0.8-4.0$ versus $1, \mathrm{IQR}=0-2.5 ; P=.40)$, and the likelihood of early clinical deterioration $(19.4 \%$ versus $4.3 \%, P=.38)$ were higher in the presence of a perfusion abnormality than without it, but these did not reach clinical significance.

Table 2 demonstrates the confidence scores for each technique, showing a reduction of equivocal studies from $24.7 \%$ with NCCT to $19.9 \%$ and $10.2 \%$ with CTA-SI and CTP, respectively. Confidence for the presence and absence of infarct increased incrementally with multimodal assessment. Significant correlation between the observed and actual diagnoses for CTA-SI and CTP modalities versus NCCT was demonstrated. Best fit was demonstrated for the multimodal approach including CTP (Table 3); progressive entropy $r^{2}$ values increased from 0.015 to 0.329 and AIC was reduced (451.1 to 357.3) with the incremental protocol. The observed diagnosis was significantly related to the actual diagnosis after adjusting for confidence level for CTP $(P=.04)$ and was 5.1 and 2.4 times more likely to confirm LI than NCCT or 
Table 3: Progressive increase of multiple entropy $r^{2}$ value and decrease of AIC with the incremental protocol ${ }^{\mathrm{a}}$

\begin{tabular}{|c|c|c|c|c|c|c|}
\hline & \multicolumn{4}{|c|}{ Logistic Regression Model } & \multicolumn{2}{|c|}{ GEEs Method } \\
\hline & $r^{2}$ & AIC & $P$ Value & Odds Ratio $(95 \% \mathrm{Cl})$ & AIC & $P$ Value \\
\hline \multicolumn{7}{|l|}{ NCCT } \\
\hline Model fit statistics & 0.015 & 451.1 & & & 451.3 & \\
\hline Observed diagnosis (yes vs no) & & & .28 & $1.68(0.65-4.41)$ & & .29 \\
\hline Observed confidence score & & & .90 & $1.03(0.67-1.58)$ & & .90 \\
\hline \multicolumn{7}{|l|}{$\mathrm{NCCT}+\mathrm{CTA}-\mathrm{SI}$} \\
\hline Model fit statistics & 0.170 & 409.2 & & & 408.7 & \\
\hline Observed diagnosis (yes vs no) & & & .59 & $0.78(0.31-1.94)$ & & .57 \\
\hline Observed confidence score & & & $<.001^{\mathrm{b}}$ & $0.46(0.31-0.68)$ & & $<.001^{\mathrm{b}}$ \\
\hline \multicolumn{7}{|l|}{$\mathrm{NCCT}+\mathrm{CTA}-\mathrm{SI}+\mathrm{CTP}$} \\
\hline Model fit statistics & 0.329 & 357.3 & & & 357.1 & \\
\hline Observed diagnosis (yes vs no) & & & $.04^{\mathrm{b}}$ & $0.33(0.11-0.95)$ & & .05 \\
\hline Observed confidence score & & & $<.001^{\mathrm{b}}$ & $0.30(0.20-0.44)$ & & $<.001^{\mathrm{b}}$ \\
\hline
\end{tabular}

Note:-GEEs indicates generalized estimating equations.

a With logistic regression analysis and the GEEs method, the actual stroke diagnosis was modelled on different observed diagnoses (NCCT alone, NCCT + CTA-SI, NCCT + CTA-SI + CTP) when adjusting for the corresponding confidence score. OR $<1$ indicates that patients with a positive diagnosis on MRI are more likely to have a lower level of confidence $(1=$ definitely present, 2 = probably present, $3=$ possibly present, $4=$ possibly absent, $5=$ probably absent, $6=$ definitely absent).

b Statistically significant.

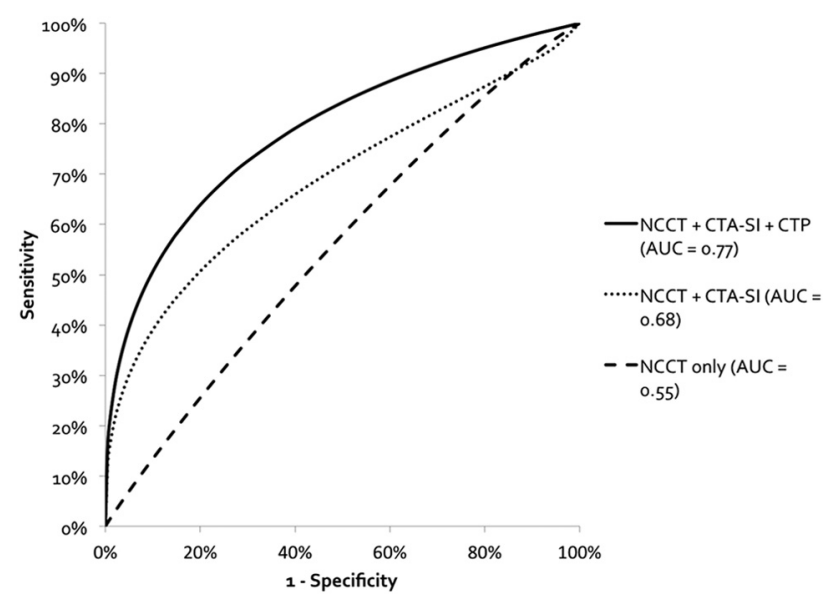

FIG 1. Receiver operating characteristic curves show the diagnostic performance of the incremental CT protocol in the detection of lacunar infarction, without adjusting for multiple readers. There is a statistically significant increase in the area under the curve with each incremental protocol (CTP versus CTA, $P<.006$, and CTP versus NCCT, $P<.001)$. The dashed line indicates NCCT only; the dotted line, NCCT and CTA-SI; and the solid line, NCCT, CTA-SI, and CTP.

CTA-SI, respectively. Incremental protocol use was associated with a correlation coefficient increase of -0.09 (95\% CI, $-0.20-$ $0.01 ; P=.08),-0.35$ (95\% CI, -0.44 to $-0.26 ; P<.001)$, and -0.48 (95\% CI, -0.55 to $-0.39 ; P<.001)$. When one considers the 3 CTP maps separately, MTT demonstrated the best model fit and overall performance $\left(r^{2}=0.320\right.$, AIC $\left.=360.3\right)$, while CBV performance was the poorest $\left(r^{2}=0.265\right.$, AIC $\left.=378.0\right)$.

Receiver operating characteristic analysis (Fig 1) demonstrated higher area under the curve for CTP $(0.77 ; 95 \%$ CI, $0.72-$ $0.81)$ than CTA-SI $(0.68 ; 95 \%$ CI, $0.63-0.73 ; P=.006)$ or NCCT alone $(0.55 ; 95 \% \mathrm{CI}, 0.50-0.60 ; P<.001)$. The difference between CTA-SI and NCCT alone was also statistically significant $(P<.001)$.

Diagnostic performance improved with incremental protocol use (Table 4). Although interobserver agreement increased with the incremental protocol (poor-to-moderate), this was not statistically significant (Table 5).

\section{DISCUSSION}

Our results demonstrate a significantly improved area under the curve and confidence for correct diagnosis, with fewer equivocal scan outcomes by using a multimodal approach. Similarly, increased interobserver reliability is demonstrated compared with conventional NCCT with a fair overall performance.

A recent study evaluating the efficacy of a newly established incremental CTP protocol found that 10\% of false-negative CTP studies in patients with clinical stroke were attributable to LI. ${ }^{12}$ The false-negative rate is multifactorial and reflects a learning curve in LI CTP pattern identification ${ }^{2,16,25}$ but also variability in the size of perfusion abnormality in the context of LI. ${ }^{15}$ Traditionally, studies have focused on identifying larger hemispheric perfusion defects with or without concurrent deep abnormalities. In the absence of cortical involvement, pure LIs are often overlooked because of their smaller size and inattention to a typical lacunar pattern of perfusion abnormality within the perforator territories including brain stem, basal ganglia, and thalamus. In our experience, LIs are poorly detected with NCCT and CTA. CTP significantly improves detection but requires careful evaluation of the perforator locations for small perfusion defects in the absence of a larger perfusion abnormality (Figs 2 and 3).

The frequency and significance of perfusion abnormality in LI was previously addressed in several small series. ${ }^{26-28}$ One of the largest studies (22 patients) demonstrated MTT abnormality in $68.2 \%$ of LIs, comparing favorably with $61 \%$ in the present series. Other smaller studies report perfusion abnormality in $0 \%^{26,28}$ to $100 \%{ }^{14}$ of cases. Clinically, perfusion abnormality in LI was previously shown to be associated with the persistence of symptoms beyond 24 hours. ${ }^{13}$ In the present study, analysis of CTP-positive and CTP-negative findings of LI showed a higher NIHSS score, shorter time to scanning, and poorer outcome in patients with a perfusion abnormality. Pathophysiologic differences may explain the variability of perfusion abnormality in the context of LI. LIs associated with perfusion defects may be secondary to atherothrombotic occlusion of several perforator branches rather than single 40 - to $200-\mu \mathrm{m}$ lipohyalinotic perforator occlusions. In support, we found both hypertension and diabetes to be associated with LI overall, but neither were significantly different between 
Table 4: Diagnostic performance for stroke detection with incremental study review using receiver operating characteristic-determined thresholds for level of confidence

\begin{tabular}{|c|c|c|c|c|}
\hline & \multicolumn{2}{|c|}{ Sensitivity } & \multicolumn{2}{|c|}{ Specificity } \\
\hline & $\mathrm{Se} \%(95 \% \mathrm{Cl})$ & $P$ Value ${ }^{\mathrm{a}}$ & Sp\% $(95 \% \mathrm{Cl})$ & $P$ Value $^{\mathrm{a}}$ \\
\hline \multicolumn{5}{|l|}{ Level of confidence $\geq 5$ vs $<5$} \\
\hline 1) $\mathrm{NCCT}$ & $40.7(30.8-50.6)$ & 1 vs 2: .174 & $70.5(64.4-76.7)$ & lvs 2:.088 \\
\hline 2) NCCT + CTA-SI & $51.7(40.9-62.5)$ & 1 vs $3: .048^{\mathrm{b}}$ & $78.2(72.2-84.2)$ & 1 vs $3: .002^{b}$ \\
\hline 3) $\mathrm{NCCT}+\mathrm{CTA}-\mathrm{SI}+\mathrm{CTP}$ & $55.9(45.6-66.3)$ & 2 vs $3: .536$ & $83.3(78.3-88.3)$ & 2 vs $3: .155$ \\
\hline \multicolumn{5}{|l|}{ Level of confidence $\leq 2$ vs $<2$} \\
\hline 1) $\mathrm{NCCT}$ & $9.3(4.3-14.3)$ & 1 vs $2: .009^{\mathrm{b}}$ & $91.9(88.5-95.2)$ & 1 vs 2:.130 \\
\hline 2) NCCT + CTA-SI & $26.3(17.3-35.3)$ & 1 vs $3:<.001^{b}$ & $95.3(92.5-98.1)$ & 1 vs 3:.691 \\
\hline 3) NCCT + CTA-SI + CTP & $42.4(30.6-54.2)$ & 2 vs $3: .030^{\mathrm{b}}$ & $91.9(88.4-95.4)$ & 2 vs $3: .264$ \\
\hline
\end{tabular}

Note:-Se indicates sensitivity; Sp, specificity.

a $P$ value was obtained by a linear regression model of natural $\log (\mathrm{Se})$ or $\log (\mathrm{Sp})$ for each modality.

b Statistically significant.

Table 5: Interobserver variability between 2 readers for lacunar infarction detection with incremental protocol

\begin{tabular}{lccc}
\hline & \multicolumn{3}{c}{ Cohen $\kappa(95 \%$ CI) for Each Sequence } \\
\cline { 2 - 4 } & NCCT & NCCT + CTA-SI & NCCT + CTA-SI + CTP \\
\hline Reader 1 vs 2 & $0.25(0.11-0.39)$ & $0.47(0.34-0.61)$ & $0.50(0.35-0.64)$
\end{tabular}
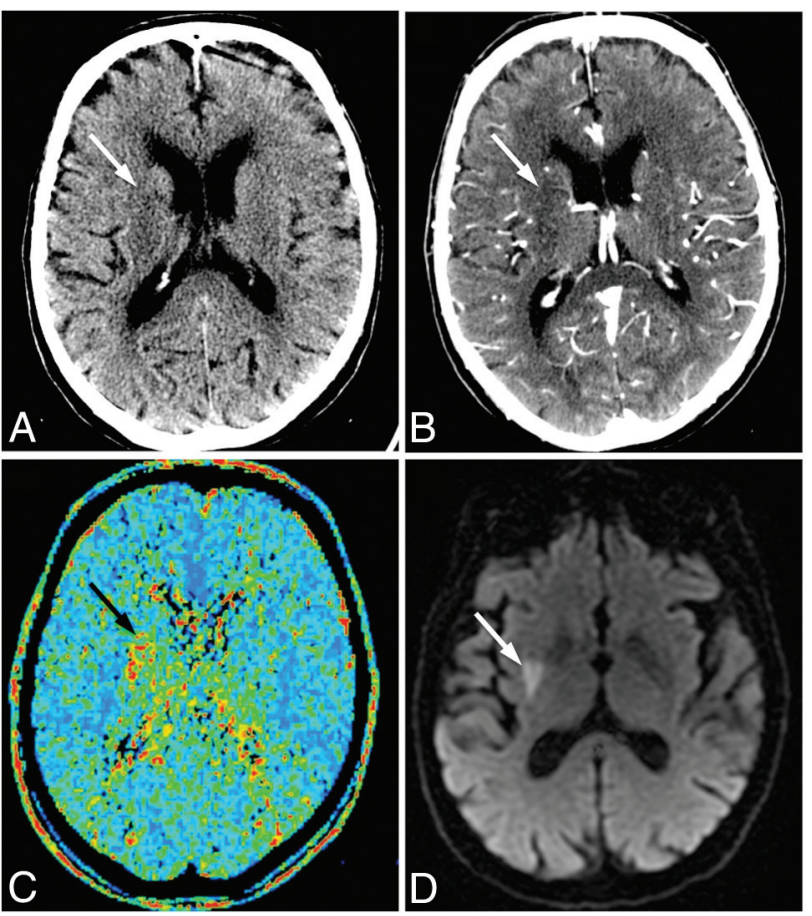

FIG 2. Images of a 72-year-old man who presented with left-sided weakness (NIHSS score, 9), scanned 85 minutes after symptom onset. NCCT $(A)$ and CTA-SI $(B)$ show subtle hypoattenuation, inconclusive for acute infarction (white arrows). C, The MTT map demonstrates a perfusion deficit in the right lentiform nucleus (black arrow). The patient received intravenous thrombolysis. D, Follow-up DWI confirms lacunar infarction in the right lentiform nucleus (white arrow).

patients with and without a perfusion defect. These results mirror a large community-based prospective cohort study of 15,792 middle-aged adults in whom both hypertension and diabetes were associated with smaller (but not large) infarcts. ${ }^{25}$

The utility of CTP in the context of LI has been previously studied. ${ }^{8,13,14}$ Early clinical deterioration occurs in $27 \%-62 \%$ of LI cases and is not reliably predicted clinically. ${ }^{26,28}$ Perfusion abnormality absence was highly predictive of clinical stability in 1
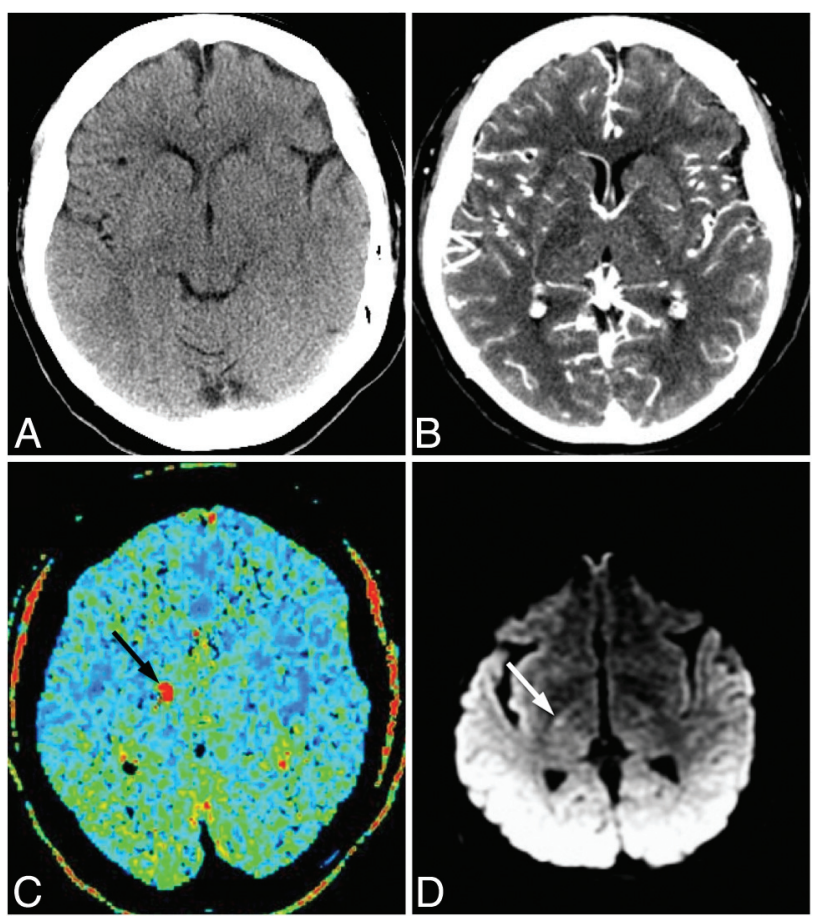

FIG 3. Images of a 55-year-old man who presented with left-sided weakness. A, No abnormality is evident on NCCT. B, CTA-SI shows a possible hypoattenuation in the posterior limb of the right internal capsule or thalamus. C, MTT map demonstrates a focus of increased MTT in the region of the internal capsule (black arrow). The patient received intravenous thrombolysis. D, Follow-up DWI confirms focal hyperintensity consistent with recent lacunar infarction in the posterior limb of the right internal capsule (white arrow).

study, while one-third of patients with perfusion abnormality experienced clinical deterioration. ${ }^{14}$ Yamada et al ${ }^{13}$ demonstrated higher MTT ratios and lower CBF ratios in patients experiencing clinical deterioration. The reported reversal of a matched right thalamostriate perfusion and diffusion abnormality following thrombolysis suggests a potential role for perfusion imaging in the management of LI. ${ }^{8}$ Although thrombolysis in LI is controversial, growing evidence supports its use. Lacunar strokes treated with IV rtPA in the National Institutes of Neurological Disorders and Stroke study demonstrated more favorable outcomes compared with a placebo group ( $63 \%$ versus $40 \%$; mRS, $\leq 1) .{ }^{4}$ Similarly, a Canadian Stroke Network Registry study of 11,503 patients showed equal benefit after thrombolysis for lacunar and total anterior circulation strokes. ${ }^{29}$ Patients with thrombolysis-treated 
ischemic stroke with small-vessel infarcts showed better clinical outcome and lower hemorrhagic complications than those secondary to other etiologies. ${ }^{30,31}$

Justifiable concern remains regarding the radiation dose from CTP, conferring an effective dose of $\sim 2.5 \mathrm{mSv}$. The total dose of the CT protocol is $6.1 \mathrm{mSv}$ by the International Commission on Radiological Protection 103 criteria. Thus, the relative incremental CTP dose is small but conferring the benefit of increased confidence of diagnosis. A recent study has shown that this could be reduced by $50 \%$ by using $50 \mathrm{~mA}$ instead of $100 \mathrm{~mA} .^{32}$ Furthermore, due to scan obliquity, no significant lens dose exposure was experienced. The largest contributor to dose remains the CTA component, due to spatial coverage and exposure to the thyroid and lenses. MR imaging in acute ischemic stroke is considered a reference standard ${ }^{33}$ and indeed provides superior infarct core delineation. Unfortunately, the overwhelming majority of tertiary stroke centers and other hospitals receiving patients with acute ischemic stroke do not have acute MR imaging access. Therefore, although MR imaging triage is recommended by consensus, this is usually after a decision to treat based on NCCT. ${ }^{33}$ We argue simply that CTA and CTP use improves initial detection over NCCT without precluding subsequent MR imaging use according to institutional resource availability. Dose is important in terms of overall population burden, and conscientious effort should be made to conform to "as low as reasonably achievable" principles and to reduce the dose in all patients. While MR imaging has the advantage of not conferring any radiation dose, the biologic effect of CT dose in an elderly population is small. ${ }^{21}$

Limitations include a retrospective study design. Although relatively small in sample size, the study does constitute one of the largest imaged lacunar cohorts. Readers were blinded to clinical information but were aware of the nature of the study; this circumstance introduced potential bias. It could also be argued that selection of particular CTP sections biases CTP detection by limiting the number of sections to a region of interest and unblinds readers. While CTP scans were directed to the posterior fossa or supratentorium by the attending neurologist, depending on the clinical presentation, standard protocol-mandated anatomic landmarks were used for every acquisition. While neuroanatomic localization may be useful in lesion detection, exact lesion determination is frequently not possible and the requirement of both a positive score and correct location reduces the possibility of bias. Prior strategies in acute stroke work-up necessitate MR imaging within a week of ictus in the context of a clinically significant vessel stenosis, placing pressure on limited and costly resources in busy acute stroke centers. A CTP study with positive findings may circumvent this need. CTP studies with negative findings will still require early MR imaging for carotid endarterectomy planning, but the number of patients requiring MR imaging should be reduced by $16 \%-33 \%$ compared with NCCT and CTA approaches, respectively. LI classification using DWI/ADC may potentially miss patients due to the known reduced DWI sensitivity for posterior fossa infarcts leading to both false-positive and -negative studies. However, the bias is systematic, applying equally to all sequences. Although the readers were still within their fellowship period, they had significant experience in stroke imaging due to high-volume exposure at a tertiary stroke center, allowing generalizability to other readers.

\section{CONCLUSIONS}

Multimodal CT including CTP has an estimated sensitivity of $42 \%$ in the detection of LI and increases the diagnostic performance 5.1- and 2.4-fold over NCCT and/or CTA-SI.

\section{ACKNOWLEDGMENTS}

We gratefully acknowledge our radiographic technicians without whom this work would not be possible.

Disclosures: Mark Boulos—UNRELATED: Other: clinical research fellowship in stroke and sleep medicine, Comments: The clinical research fellowship was funded by the Canadian Institutes of Health Research, the Heart and Stroke Foundation, and the Canadian Stroke Network. Sean P. Symons_UNRELATED: Grants/Grants Pending: Brain Tumour Foundation of Canada*; Payment for Lectures (including service on Speakers Bureaus): Biogen (MS lectures). *Money paid to the institution.

\section{REFERENCES}

1. Kolominsky-Rabas PL, Weber M, Gefeller O, et al. Epidemiology of ischemic stroke subtypes according to TOAST criteria: incidence, recurrence, and long-term survival in ischemic stroke subtypes: a population-based study. Stroke 2001;32:2735-40

2. Wardlaw JM, Smith C, Dichgans M. Mechanisms of sporadic cerebral small vessel disease: insights from neuroimaging. Lancet $\mathrm{Neu}$ rol 2013;12:483-97

3. Norrving B. Long-term prognosis after lacunar infarction. Lancet Neurol 2003;2:238-45

4. National Institute of Neurological Disorders and Stroke rt-PA Stroke Study Group. Tissue plasminogen activator for acute ischemic stroke. N Engl J Med 1995;333:1581-87

5. IST-3 collaborative group. The benefits and harms of intravenous thrombolysis with recombinant tissue plasminogen activator within $6 \mathrm{~h}$ of acute ischaemic stroke (the third International Stroke Trial [IST-3]): a randomised controlled trial. Lancet 2012;379:2352-63

6. Fuentes B, Martínez-Sánchez P, Alonso de Leciñana M, et al. Efficacy of intravenous thrombolysis according to stroke subtypes: the Madrid Stroke Network data. Eur J Neurol 2012;19:1568-74

7. Pantoni L, Fierini F, Poggesi A. Thrombolysis in acute stroke patients with cerebral small vessel disease. Cerebrovasc Dis 2014;37:5-13

8. Chalela JA, Ezzeddine M, Latour L, et al. Reversal of perfusion and diffusion abnormalities after intravenous thrombolysis for a lacunar infarction. J Neuroimaging 2003;13:152-54

9. Wessels T, Röttger C, Jauss M, et al. Identification of embolic stroke patterns by diffusion-weighted MRI in clinically defined lacunar stroke syndromes. Stroke 2005;36:757-61

10. Young KC, Benesch CG, Jahromi BS. Cost-effectiveness of multimodal CT for evaluating acute stroke. Neurology 2010;75:1678-85

11. Hopyan J, Ciarallo A, Dowlatshahi D, et al. Certainty of stroke diagnosis: incremental benefit with CT perfusion over noncontrast CT and CT angiography. Radiology 2010;255:142-53

12. Campbell BCV, Weir L, Desmond PM, et al. CT perfusion improves diagnostic accuracy and confidence in acute ischaemic stroke. J Neurol Neurosurg Psychiatry 2013;84:613-18

13. Yamada M, Yoshimura S, Kaku Y, et al. Prediction of neurologic deterioration in patients with lacunar infarction in the territory of the lenticulostriate artery using perfusion CT. AJNR Am J Neuroradiol 2004;25:402-08

14. Poppe AY, Coutts SB, Kosior J, et al. Normal magnetic resonance perfusion-weighted imaging in lacunar infarcts predicts a low risk of early deterioration. Cerebrovasc Dis 2009;28:151-56

15. Gerraty RP, Parsons MW, Barber PA, et al. Examining the lacunar 
hypothesis with diffusion and perfusion magnetic resonance imaging. Stroke 2002;33:2019-24

16. Fisher CM. Lacunes: small, deep cerebral infarcts. Neurology 1965;15:774-84

17. Lansberg MG, Thijs VN, O’Brien MW, et al. Evolution of apparent diffusion coefficient, diffusion-weighted, and T2-weighted signal intensity of acute stroke. AJNR Am J Neuroradiol 2001;22:637-44

18. Potter GM, Marlborough FJ, Wardlaw JM. Wide variation in definition, detection, and description of lacunar lesions on imaging. Stroke 2011;42:359-66

19. Doege CA, Kerskens CM, Romero BI, et al. Assessment of diffusion and perfusion deficits in patients with small subcortical ischemia. AJNR Am J Neuroradiol 2003;24:1355-63

20. Restrepo L, Jacobs MA, Barker PB, et al. Etiology of perfusion-diffusion magnetic resonance imaging mismatch patterns. J Neuroimaging 2005; 15:254-60

21. Brenner DJ, Hall EJ. Computed tomography: an increasing source of radiation exposure. $N$ Engl J Med 2007;357:2277-84

22. DeLong ER, DeLong DM, Clarke-Pearson DL. Comparing the areas under two or more correlated receiver operating characteristic curves: a nonparametric approach. Biometrics 1988;44:837-45

23. Zhou X-H, Obuchowski NA, McClish DK. Comparing the accuracy of two diagnostic tests. In: Statistical Methods in Diagnostic Medicine, 2nd ed. Hoboken: John Wiley \& Sons; 2011:165-92

24. Barnhart HX, Williamson JM. Weighted least-squares approach for comparing correlated kappa. Biometrics 2002;58:1012-19
25. Bezerra DC, Sharrett AR, Matsushita K, et al. Risk factors for lacune subtypes in the Atherosclerosis Risk in Communities (ARIC) study. Neurology 2012;78:102-08

26. Mohr JP, Caplan LR, Melski JW, et al. The Harvard Cooperative Stroke Registry: a prospective registry. Neurology 1978;28:754-62

27. Nakamura K, Saku Y, Ibayashi S, et al. Progressive motor deficits in lacunar infarction. Neurology 1999;52:29-33

28. Tei $\mathrm{H}$, Uchiyama $\mathrm{S}$, Ohara $\mathrm{K}$, et al. Deteriorating ischemic stroke in 4 clinical categories classified by the Oxfordshire Community Stroke Project. Stroke 2000;31:2049-54

29. Shobha N, Fang J, Hill MD. Do lacunar strokes benefit from thrombolysis? Evidence from the registry of the Canadian Stroke Network. Int J Stroke 2013;8(suppl A100):45-49

30. Mustanoja S, Meretoja A, Putaala J, et al. Outcome by stroke etiology in patients receiving thrombolytic treatment: descriptive subtype analysis. Stroke 2011;42:102-06

31. Lee SJ, Saver JL, Liebeskind DS, et al. Safety of intravenous fibrinolysis in imaging-confirmed single penetrator artery infarcts. Stroke 2010;41:2587-91

32. Murphy A, So A, Lee TY, et al. Low dose CT perfusion in acute ischemic stroke. Neuroradiology 2014;56:1055-62

33. Wintermark M, Sanelli PC, Albers GW, et al. Imaging recommendations for acute stroke and transient ischemic attack patients: a joint statement by the American Society of Neuroradiology, the American College of Radiology, and the Society of NeuroInterventional Surgery. AJNR Am J Neuroradiol 2013;34:E117-27 